Résumés des conférences et travaux

\title{
Jean-Pierre Diény (1927-2014)
}

\section{François Martin}

\section{OpenEdition \\ Journals}

Édition électronique

URL : https://journals.openedition.org/ashp/1790

DOI : 10.4000/ashp.1790

ISSN : 1969-6310

Éditeur

Publications de l'École Pratique des Hautes Études

Édition imprimée

Date de publication : 1 septembre 2016

Pagination : vi-viii

ISSN : 0766-0677

Référence électronique

François Martin, « Jean-Pierre Diény (1927-2014) », Annuaire de l'École pratique des hautes études (EPHE), Section des sciences historiques et philologiques [En ligne], 147 | 2016, mis en ligne le 08 septembre 2016, consulté le 06 juillet 2021. URL : http://journals.openedition.org/ashp/1790 ; DOI : https://doi.org/10.4000/ashp.1790 


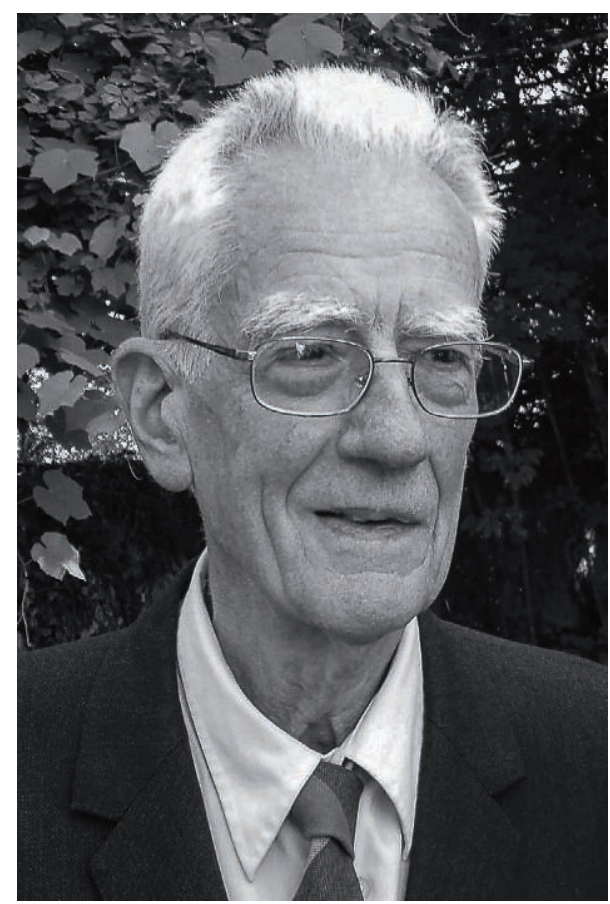

\section{JEAN-PIERRE DIÉNY}

$(1927-2014)$

$\mathrm{J}$

EAN-Pierre Diény nous a quittés le 3 mai dernier. Il était né le 14 août 1927 à Colmar. Reçu premier à l'agrégation de lettres classiques, il enseigna le grec de 1952 à 1955 au lycée Fustel de Coulanges de Strasbourg. Il avait cependant déjà pris la résolution de se consacrer à l'étude de la civilisation chinoise dont la révélation lui avait été donnée tout d'abord dans la bibliothèque de l'École normale par des livres sur l'art chinois. Cette découverte devait éveiller son intérêt pour la Chine et décider de toute une vie de recherches. Mais surtout, il avait fait la connaissance de Paul Demiéville, grand maître des études chinoises, qui enseignait alors à la section, et qui cherchait un répétiteur pour son fils. Ce fut la chance d'une vie. S'ouvrit alors entre les deux hommes une relation privilégiée, faite de respect et d'admiration mutuelle. Jean-Pierre Diény dira de Paul Demiéville : « Jamais envers aucun maître je n'ai contracté une telle dette de reconnaissance ».

L'obtention, en 1955, d'une bourse de la fondation Thiers, va lui permettre de se consacrer à l'étude de la littérature chinoise. Il sera nommé attaché de recherches au CNRS la même année. L'autre grande chance de sa vie, la plus grande selon lui, fut la rencontre de Colette Patte (1924-2013), qu'il épouse en 1958. Ils vivront ensemble 
leur passion commune; Colette s'est spécialisée dans l'étude de l'histoire des sciences en Chine et l'histoire des jésuites. Sa vie durant, son époux l'encouragera à poursuivre ses travaux.

En 1959, Jean-Pierre Diény, nommé pensionnaire de la maison franco-japonaise de Tokyo, part avec son épouse et leur premier enfant pour le Japon, où la sinologie est alors à son zénith. Après avoir séjourné à Kyôto auprès du grand maître des études classiques chinoises, Kawakatsu Yoshio 川勝義雄, il revient à Tokyo en septembre 1962 avant de quitter le Japon en novembre. Après deux années passées en France, il arrive enfin à Pékin en novembre 1964, et y enseigne le français à l'Institut des langues étrangères. Hélas, les conditions y sont difficiles : à un enseignement lourd s'ajoute une ambiance politique oppressante; les contacts sont malaisés, l'accès aux bibliothèques difficile. La crise éclate fin 1966 : les chercheurs étrangers sont contraints à quitter la Chine. Jean-Pierre Diény s'installe à Hong Kong. C'est un mal pour un bien, car il découvre là un milieu de savants chinois, souvent des émigrés, ouvert et accueillant, et en particulier il se lie à Rao Zongyi 饒宗頣, un des plus grands érudits chinois de notre temps.

De retour en France avec son épouse et leurs quatre enfants - le plus jeune vient de naître, à Hong Kong, il est promu chargé de recherches au CNRS. Dès 1965, son livre Dix-neuf poèmes anciens, publié au Japon, devenu un classique à l'étranger comme en France, lui avait valu le diplôme de l'EPHE. Et en 1969, il reçoit le prix Giles de l'Académie des inscriptions et belles-lettres pour le livre Aux origines de la poésie chinoise classique. Chargé de conférences à la IV ${ }^{\mathbb{E}}$ section en 1969, il est élu directeur d'études en 1970. Il enseignera à la section jusqu'en 1997.

Dans les rapports qu'il a été amené à rédiger sur son disciple, Paul Demiéville n'a jamais manqué de souligner la rarissime convergence chez Jean-Pierre Diény d'une formation classique rigoureuse et de la double formation chinoise et japonaise; sa modestie l'empêchait évidemment de mentionner son propre enseignement, le meilleur de ce que pouvait apporter alors la sinologie occidentale.

L'héritage scientifique de Jean-Pierre Diény est considérable. Par ses très nombreuses publications, ouvrages, articles, des comptes rendus aussi précieux que des études, en français pour l'essentiel, mais parfois aussi en japonais et en chinois, il aura beaucoup œuvré pour le rayonnement de l'École. S'il savait saluer les grandes synthèses, il leur préférait, fidèle à l'esprit de l'École, une méthode prudente et sûre, fondée sur l'examen minutieux des sources. Il disait lui-même voir la civilisation de la Chine ancienne comme un continent encore peu connu, dans lequel il ne fallait progresser qu'avec précaution. Sa connaissance était comme un édifice qu'il fallait construire pierre à pierre. Une vie d'homme ne pouvait suffire à achever l'édifice, mais il en aura laissé à ceux qui voudront poursuivre son œuvre les bases saines et solides.

Jean-Pierre Diény rêvait d'un lexique de l'imagination littéraire en Chine et savait interroger ces thèmes et ces images qui fondent les civilisations, et qu'une familiarité trompeuse porte à mésinterpréter, voire à négliger. Ainsi du dragon et du phénix, qu'il sut piéger dès leur apparition dans les documents les plus anciens, et suivre dans leur vol majestueux au long des siècles, leur rendant une vie qui avait été si féconde dans les siècles lointains et nous interdisant désormais de n'y voir que des décors de restaurant chinois. Son Symbolisme du dragon dans la Chine antique est un modèle du 
genre. Parfois encore, c'était la recherche autour d'une œuvre, ou autour d'un homme, comme celle qui aboutit au si beau Portrait anecdotique d'un gentilhomme, Xie An (320-385) dans le Shishuo xinyu, hommage d'un gentilhomme d'aujourd'hui à un gentilhomme d'autrefois.

Ces recherches donnaient lieu à des enquêtes passionnantes. On se pressait à ses conférences, et il est peu de sinologues des générations suivantes qui n'ont pas suivi son enseignement, et parfois pendant de longues années.

Le départ à la retraite ne fut point pour lui la fin des activités, mais le début d'un otium fécond. Tout en menant à leur terme bien des travaux encore non aboutis, il se fit plaisir - c'étaient ses mots - en publiant un florilège de traduction de poèmes paysagers chinois, Jeux de montagne et d'eaux. Il y retrouvait l'enthousiasme de ses premières découvertes de jeunesse et donnait libre cours à son amour pour la montagne, qu'il partageait avec son maître. Il l'avait enfin découverte en Chine lors d'un voyage accompli en 1986, et parcouru en d'interminables randonnées, qu'il aimait à évoquer. Mais s'il se faisait ainsi plaisir à lui-même, il en donnait aussi beaucoup aux autres. La métrique irréprochable et la langue élégante font de cet ouvrage un chef-d'œuvre de l'art de la traduction.

Enfin, il anima longtemps un petit groupe de volontaires accomplissant à la Bibliothèque de l'Institut des hautes études chinoises un travail ingrat mais précieux de rangement, de classement et de mise en fiches.

Mais vinrent la vieillesse, l'épuisement, la maladie. La mort de sa chère épouse, le jour de Noël 2013, lui porta le dernier coup et lui ôta toute volonté de vivre. Il dut accepter de s'installer dans une maison de retraite à Thonon, près de deux de ses enfants, et en vue des montagnes. C'est là qu'il s'est éteint le 3 mai dernier.

Homme imprégné de religion - il disait volontiers qu'il aurait pu être pasteur - il a laissé à tous ceux qui l'ont connu le souvenir d'un homme modeste et discret, mais chaleureux, généreux et attentif aux autres.

$$
\text { François MARTIN (†) }
$$

Nota : une bibliographie des travaux de Jean-Pierre Diény a été publiée dans le second volume des Mélanges qui lui ont été offerts en 1999-2000, p. 325-332. Voir François Martin (dir.), Mélanges de sinologie offerts à Monsieur Jean-Pierre Diény, vol. I, Études chinoises, vol. XVIII, 1-2, printemps-automne 1999 (vol. I) et vol. XIX, no 1-2, printemps-automne 2000 (vol. II). La liste des thèmes abordés par Jean-Pierre Diény lors de ses conférences à l'EPHE figure dans le vol. II, p. 337-340. Deux livres sont parus depuis. Jean-Pierre Diény a assuré l'édition scientifique du premier, en plus de ses propres traductions : Ce dont le Maître ne parlait pas. Le merveilleux onirique, par Yuan Mei, récits traduits du chinois, présentés et annotés par Chang Fu-Jui, Jacqueline Chang, Jean-Pierre Diény, Paris, Gallimard, NRF, 2011, 369 p. Le second livre est un recueil comprenant avec des révisions et des compléments presque tous ses articles et contributions. Cet ouvrage a été édité avec le plus grand soin par Pierre-Henri Durand, directeur de recherches au CNRS, en étroite collaboration avec Jean-Pierre Diény, Images et représentations du monde dans la Chine ancienne. Choix d'études (1962-2006), 2 tomes, Paris, Collège de France, Institut des hautes études chinoises, 2012, 673 p. [Alain Thote] 